\title{
Effects of dust on soil changes: from tropical islands to alpine meadow in the east Eurasia
}

\author{
GAN-LIN ZHANG ${ }^{1 *}$, FEI YANG ${ }^{1}$
}

${ }^{1}$ State Key Laboratory of Soil and Sustainable Agriculture, Institute of Soil Science, Chinese Academy of Sciences, Nanjing, China (*Correspondence: glzhang@issas.ac.cn)

Dust deposition occurs across the world but in different amplititude and impacts. Its effects on terrestrial ecosystems vary greatly depending on dust flux and composition, substrate geology and climate of the receiving ecosystem. Typical areas in China including the tropical Hainan Island in the south, northeastern Qinghai-Tibet Plateau (NE-QTP) in the northwest and Nanjing, a metropolis urban area in the east were studied, representing dust from natural and anthropogenic sources and different climate, vegetation and original soils. The NE-QTP is mainly controlled by winter monsoon and westerlies while Hainan mainly by summer monsoon and Nanjing alternatively by both of them with seasonal changes.

Our study reveals that loess deposition in high fluxes since the Holocene in NE-QTP is the major source for the silty surface soils, which is genetically independed on the underlying debris. Following the elevation gradient and accompanied rainfall and vegetation changes, secondary soil development processes vary fundamentally and determine soil physicochemical properties and thus major soil functions, such as soil water holding capacity and soil carbon accumulation. Current dry deposition in the Hainan Island is very low, however our study shows historical continental dust deposition had a persisting effect on soil properties. This process is evidenced by $\mathrm{Sr}$ and $\mathrm{Nd}$ isotope shift of soils sequentially developed on basalt of different ages. Dust may have provided mineral nutrients to this tropical ecosystem. The urban area in the east China receives both naturally transported loess and anthropogenically produced industrial dust, as well as oceanic input. It is phenomenal that pollutants carried by dust of local and remote sources are accumulated in surface soil, showing the potential ecological risks induced by modern dust inputs.

Considering the gegraphical extent and the spatialtemporal variation in flux and composition, dust deposition has changing but important impacts on typical ecosystems in the east part of Eurasia continent. A better assessment of the overall impacts on terrestrial ecosystem develpoment and functioning is necessary. 